\title{
Análisis de las concepciones de los alumnos en torno a una experiencia sobre desarrollo y crecimiento de la Drosophila melanogaster
}

\section{Analysis of Students' Conceptions about an Experience on Development and Growth of Drosophila Melanogaster}

Grupo de investigación: Biología, Enseñanza y Realidades

\author{
Gerardo Ruiz Sánchez ${ }^{1}$ \\ Jhon Jairo Martínez Murillo \\ Sandra Ximena Ibáñez Córdoba ${ }^{3}$ \\ Guillermo Chona Duarte ${ }^{4}$
}

Fecha de recepción: 1 de abril de 2016

Fecha de aprobación: 13 de octubre de 2016

\section{Resumen}

En esta investigación se analizan las concepciones presentes en el discurso de alumnos de octavo grado del Colegio Ciudad de Bogotá, Institución Educativa Distrital, en torno al desarrollo y el crecimiento, a través de la experiencia con el ciclo de vida de Drosophila melanogaster. La investigación se dividió en dos momentos: en el primero, a través de una encuesta, se identificaron las concepciones iniciales de los alumnos, y en el segundo, los alumnos, junto con el docente, trabajaron in situ el ciclo de vida del insecto objeto de estudio. Las interacciones discursivas fueron grabadas en audios, transcritas y categorizadas; posteriormente, se las analizó desde el nivel semántico de acuerdo con los planteamientos de Teun van Dijk (1985). Con los significados asignados, se establecieron implicaciones sobre la enseñanza del contenido de estudio en ciencias naturales. El método de la investigación consideró: la perspectiva cualitativa-interpretativa, el enfoque del análisis del discurso y el empleo de las técnicas de codificación abierta - categorización- para el primer momento, y de análisis semántico, para el segundo momento. El proceso investigativo facilitó que los alumnos resignificaran sus saberes mediante la experiencia de aula; es decir, sus concepciones iniciales fueron transformándose en la medida en que confrontaban el ciclo de vida de Drosophila melanogaster con la información adquirida a través de la interacción discursiva.

Palabras clave: concepciones, crecimiento, desarrollo, discurso, semántica, significados, enseñanza de la biología

\section{Abstract}

This research analyses eighth-grade students' conceptions of development and growth in the light of their experience with the life cycle of Drosophila melanogaster, at Ciudad de Bogotá School. This study was carried out in two stages: in the first, an exploratory survey was applied to identify the initial students' conceptions, and in the second stage of the study, teachers worked the insect life

1 Docente de Ciencias Naturales y Educación Ambiental, Colegio Ciudad de Bogotá I. E. D. Bogotá, D.C. Colombia

2 Docente de Ciencias Naturales y Educación Ambiental, Colegio Liceo Femenino Mercedes Nariño I. E. D. Bogotá, D.C. Colombia

3 Profesora-investigadora Universidad Pedagógica Nacional (Colombia) - ximenaic@yahoo.com

4 Profesor Investigador Universidad Pedagógica Nacional (Colombia) - guichodu@yahoo.com 
cycle with the students in situ. Their discursive interactions were recorded, transcribed, categorized, and subsequently analyzed, according to semantic discourse analysis proposed by Teun van Dijk (1985). The implications on teaching the Natural Sciences contents were established by using the assigned meanings. The research method involved a qualitative-interpretative perspective; the approach of discourse analysis and the use of open coding techniques -categorization- in the first phase, and the semantic analysis for the second one. This research methodology allowed students to re-signify their knowledge, through the classroom experience, that is to say, students initial conceptions were transformed as they were confronting the Drosophila melanogaster life cycle with the information provided by the discursive interaction.

Keywords: conceptions, growth, development, discourse, semantics, meanings, teaching of biology

\section{Introducción}

Las concepciones son el conjunto de ideas coordinadas e imágenes coherentes explicativas que utilizan los alumnos, a partir de su propia experiencia, para razonar ante situaciones problema, y que constituyen un elemento motor en la construcción del saber siendo resignificadas por el alumno con más experiencias y al originar nuevas concepciones (Giordan y De Vecchi, 1995); sin embargo, el aprendizaje no sería productivo si no se proponen esas concepciones a través de discusiones, y es en este momento cuando alumnos y docentes, mediante el discurso, construyen conocimiento basados en la interacción social y natural. En ese orden de ideas, la presente investigación, con perspectiva interpretativa, analiza las concepciones en el discurso de 38 alumnos de octavo grado del Colegio Ciudad de Bogotá, I. E. D., en dos momentos: en el primero, manifestaron sus concepciones en torno al crecimiento y el desarrollo de un organismo, y en el segundo, mediante la experiencia con Drosophila melanogaster, dieron cuenta del desarrollo y el crecimiento estudiando su ciclo de vida; en ambos casos, la sistematización permitió a los investigadores su respectivo análisis para, finalmente, establecer implicaciones en el ejercicio de la docencia respecto a la enseñanza del contenido de estudio.

Todo el anterior proceso surge de dos cuestionamientos: ¿qué significados presentan las concepciones en el discurso de los alumnos de octavo grado sobre desarrollo y crecimiento, a través de su experiencia con el ciclo de vida de Drosophila melanogaster?, y ¿qué implicaciones se pueden establecer sobre la enseñanza del saber en referencia, a partir de estos significados? En consecuencia, la investigación tuvo como propósitos centrales:

1. Identificar las concepciones iniciales que tiene el grupo de alumnos de octavo grado acerca del desarrollo y el crecimiento de los organismos.
2. Desarrollar con los alumnos una experiencia de aula utilizando el ciclo de vida de la mosca de la fruta (Drosophila melanogaster) que permita discutir sobre eventos de su desarrollo y su crecimiento.

3. Atribuir significados a las concepciones en el discurso de los alumnos generadas en la experiencia con Drosophila melanogaster aplicando reglas desde el análisis semántico.

4. Establecer implicaciones en torno a la enseñanza del asunto objeto de estudio a partir de la interpretación hecha a las concepciones en el discurso de los alumnos.

Es pertinente, por lo planteado, referenciar el sustento teórico basado en la semántica del discurso, el desarrollo y el crecimiento de los organismos y la experiencia del alumno en su aprendizaje.

\section{Referentes teóricos}

\section{La semántica del discurso}

Un referente fundamental para la etapa de análisis fue tomar en consideración al lingüista van Dijk (1985, 2007), cuyos planteamientos determinan que un estudio adecuado de las relaciones entre el discurso y la sociedad puede comenzar por un análisis de tipo semántico, donde se define el análisis de un discurso como la atribución de significados intencionales a las proposiciones que contiene, y en el cual este significado es un objeto semántico. Teun van Dijk plantea que para atribuir significados se deben tener en cuenta los principios de funcionalidad, estructuralidad y extensionalidad o referencialidad, aplicados de manera integrada, como también la coherencia semántica, todos los cuales son definidos en la figura 1.

\section{Consideraciones sobre desarrollo y crecimiento}

Para los antiguos, la reproducción fue objeto de especial interés; tanto así, que Aristóteles (384-322 a. C.) adujo, mediante su teoría de la generación espontánea, que las especies se generaban a partir de la materia inanimada. Pasaron casi dos milenios hasta cuando hombres de ciencia como Redi (1668) y Spallanzani (1769) trataron de demostrar lo inviable de dicha teoría, pero fue el científico Louis Pasteur (1860) quien, mediante sus famosos experimentos con los frascos cuello de cisne, la destruyó contundentemente. Desde esa época a la fecha, se sabe que la vida proviene de la misma vida; en consecuencia, reproducirse es una función común a todos los seres vivos: se trata del proceso por el cual los seres vivos dan 


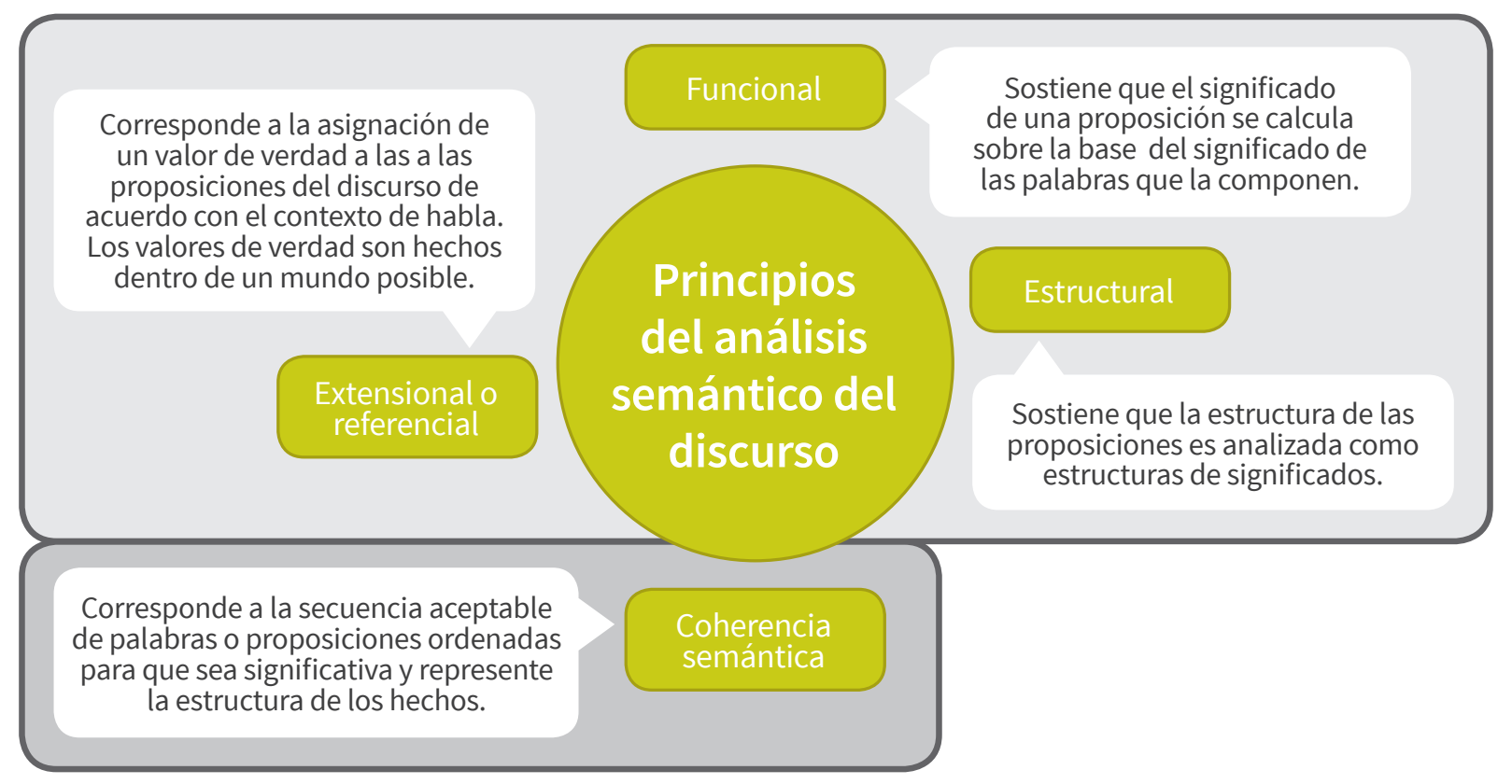

Figura 1. Reglas del análisis semántico para atribuir significados a las proposiciones de un discurso, según van Dijk (1985, 2007).

origen a nuevos individuos parecidos a ellos mismos, siguiendo una información genética (Enciclopedia del estudiante, 2006).

\section{Modalidades de reproducción}

\section{Reproducción asexual}

es el tipo de reproducción en la que se generan descendientes con características hereditarias idénticas a partir de un solo progenitor (Starr y Taggart, 2004). En los organismos unicelulares, los tipos de reproducción celular pueden ser por: bipartición, gemación, pluripartición o esporulación. Por otro lado, los tipos de reproducción asexual en animales se representa en: gemación, escisión y regeneración. La mayoría de los tipos de reproducción asexual mencionados se llevan a cabo por mitosis, que es, además, el proceso responsable del crecimiento, la regeneración y el reemplazo celular en un organismo multicelular individual de cualquier tipo, ya se reproduzca el organismo por métodos sexuales o asexuales (Kimball, 2001).

\section{Reproducción sexual}

proceso más complejo que la reproducción asexual; aun así, prácticamente todos los eucariotas lo emplean. En este tipo de reproducción participan dos progenitores de distinto sexo, cada uno de los cuales aporta un gameto con la mitad del número de cromosomas, de tal suerte que en la fecundación se restablece el número total de cromosomas pertenecientes a la especie. Los gametos se forman por la meiosis; este proceso comienza con una célula diploide $-2 n$ cromosomas $-y$ finaliza con células haploides $-\mathrm{n}$ cromosomas-.

\section{El desarrollo}

el desarrollo embrionario se inicia con la fecundación, que es la unión del gameto femenino con el masculino -ambos, haploides-, para formar un cigoto diploide. La fecundación activa al óvulo para que este desencadene cambios metabólicos que inician el desarrollo embrionario (Campbell, Mitchell y Reece, 2001); es decir, es el comienzo de una serie de cambios complicados y perfectamente organizados que originan, con el tiempo, un nuevo individuo adulto de la especie. Se utiliza el término desarrollo para describir esos cambios (Kimball, 2001). El desarrollo real es continuo y fluido; sus etapas iniciales son: segmentación, gastrulación, organogénesis y crecimiento, y tienen lugar durante la vida del embrión, en la cual se forman casi todos los órganos. Una vez nace el organismo, si sobrevive, sigue creciendo, alcanza la madurez sexual, se reproduce, envejece y, finalmente, muere. El desarrollo se puede clasificar en directo e indirecto: en el primero, los recién nacidos parecen adultos en miniatura, pero son sexualmente inmaduros - caracoles de tierra, reptiles, aves y mamíferos-. En el segundo caso, el animal joven difiere de manera significativa del adulto y experimenta cambios radicales durante el desarrollo - la mayoría de los invertebrados, incluidos los insectos y los equinodermos, y entre los vertebrados, los anfibios- (Audesirk, Audesirk y Byers, 2008). 


\section{Crecimiento y desarrollo de la mosca Drosophila melanogaster}

el desarrollo embrionario de Drosophila melanogaster comienza a partir de la fecundación del huevo; el cigoto se divide por mitosis ordenada, y, repetitivamente va originado un blastodermo sincicial (Curtis, Barnes, Schnek y Massarini, 2011). Poco después se forma el blastodermo celular, cuando la membrana que recubre el huevo se invagina rodeando cada núcleo y forma células individuales; gradualmente, las células se reubican para originar las capas embrionarias y dar paso a la gastrulación. En las etapas finales de la gastrulación aparecen una serie de intervalos y muescas regulares, que evidencian la subdivisión del cuerpo en parasegmentos, los cuales, a su vez, originarán los segmentos de la larva y del adulto. Al proceso de formación de unidades repetitivas se le conoce con el nombre de segmentación. El diseño corporal que se forma es, básicamente, el mismo en el embrión, la larva y el adulto. Cuando la mosca adulta termina el estado de pupa, el macho y la hembra presentan características corporales diferentes que acentúan su dimorfismo sexual (Curtis et al., 2011).

\section{La experiencia del alumno en su educación}

La enseñanza de las ciencias en los niños está directamente relacionada con la vivencia de su entorno; según Candela (1991), el niño, al razonar sobre los fenómenos naturales que lo rodean y tratar de explicarse sus causas, pretende que evolucionen sus condiciones, pero, sobre todo, que se desarrollen su actitud científica y su pensamiento lógico. La expresión de los saberes del niño ante sus compañeros y ante el maestro en el aula desemboca en una práctica discursiva que se convierte en un recurso metodológico, el cual, a su vez, posibilita la construcción de conocimiento o la complejización de ideas desde el lenguaje; en este proceso de construcción conjunta de significados hay un encuentro de argumentaciones negociadas (Candela, 1991), que posibilitan el análisis discursivo por parte del investigador. En el mismo sentido, para Arcá, Guidoni y Mazzoli (1990) es, pues, extremadamente difícil - casi imposible - pensar que cosas al parecer tan diversas y modos de vida tan lejanos entre sí son, en realidad, experiencias, lenguajes y conocimientos nacidos de algo que constituye la relación global entre el hombre y el mundo que lo rodea. Es partir del nivel de la experiencia, a través de un lenguaje hecho de palabras y de representaciones $-y$ sin lenguaje no sería posible-, como se puede, por tanto, construir y controlar algo - algo a lo que llamamos conocimiento-.

En este caso concreto del análisis de las concepciones en el discurso de los alumnos, el papel del docente es imprescindible, ya que, ante todo, es él quien desarrolla una verdadera competencia de estimulador de problemas y de indagador de estructuras de pensamiento, de modo que, a partir de una experiencia vivida en clase, o bien, remitiéndose a una experiencia que los jóvenes tengan en común, se llegue a una sistemática explicitación de los esquemas de explicación. Según Arcá et al. (1990), esta fase del trabajo es, para todos los efectos, un momento central y condicionante en el proceso. En efecto, es, precisamente, a partir de esas discusiones, articuladas en torno a una búsqueda de explicación coherente de hechos diversos, en variados contextos, como puede resultar posible utilizar significativamente modelos e instrumentos cognitivos capaces de reorganizar los conocimientos ya existentes en los jóvenes, y de estimular significativos desarrollos de conocimientos a través de la adquisición de nuevas experiencias y nuevos modelos.

\section{Metodología}

Dentro del marco de la investigación para realizar el análisis del contenido semántico del discurso sobre desarrollo y crecimiento a partir de la experiencia con Drosophila melanogaster, se tuvo en cuenta un grupo mixto de 38 alumnos de grado octavo, con edades entre los 12 y los 15 años, pertenecientes al Colegio Ciudad de Bogotá, I. E. D.

La investigación tiene una perspectiva interpretativa, con el enfoque de análisis del discurso y empleando la técnica de análisis de contenido semántico desde los principios y las reglas propuestas por van Dijk, $(1985,2007)$ en relación con la funcionalidad, la estructuralidad, la referencialidad y la coherencia del discurso de los alumnos -escritos, dibujos y audios- para la atribución de significados; en consecuencia, se utilizaron instrumentos como: bitácora del alumno - guías-, videos, cultivos de Drosophila melanogaster, grabaciones de discusiones en clase y material visual escaneado. La investigación se planeó en dos momentos: el momento 1 , denominado Identificación de las concepciones iniciales de los alumnos, y el momento 2, denominado Experiencia con Drosophila melanogaster.

\section{Momento 1. Identificación de las concepciones iniciales de los alumnos}

El propósito fue identificar las concepciones iniciales que los alumnos tenían respecto al desarrollo y el crecimiento de un organismo. Para tal efecto, a cada alumno se le entregó una guía, en la cual se formularon cuatro preguntas específicas; cada una con su intencionalidad (tabla 1). 
Tabla 1. Sobre las preguntas y su intencionalidad

\begin{tabular}{|l|l|}
\hline \multicolumn{1}{|c|}{ Pregunta } & \multicolumn{1}{c|}{ Intencionalidad } \\
\hline $\begin{array}{l}\text { Mediante un dibujo, represente lo que piensa cuando se le pregunta sobre } \\
\text { crecimiento y desarrollo de un organismo - explicar con palabras-. }\end{array}$ & $\begin{array}{l}\text { Interpretar las diferentes concepciones sobre crecimiento y desa- } \\
\text { rrollo que tienen los alumnos. }\end{array}$ \\
\hline $\begin{array}{l}\text { Algunas veces al partir una guayaba (fruta) nos pueden aparecer uno o } \\
\text { varios pequeños gusanos. ¿De dónde cree se han originado estos pequeños } \\
\text { gusanos? }\end{array}$ & Identificar el origen de un organismo a partir de otro preexistente. \\
\hline $\begin{array}{l}\text { Si dejamos una guayaba (fruta) al ambiente, notamos que llegan moscas. } \\
\text { ¿Para qué cree que la mosca se posará sobre la guayaba? }\end{array}$ & $\begin{array}{l}\text { Complementar la anterior pregunta, de modo que el alumno reco- } \\
\text { nozca que el gusano tiene su origen a partir de otro organismo. }\end{array}$ \\
\hline $\begin{array}{l}\text { Mediante un dibujo represente lo que ha sucedido antes y sucederá después } \\
\text { con el gusano que ha observado en la guayaba - explicar con palabras-. }\end{array}$ & $\begin{array}{l}\text { Indagar si el alumno conoce los estados de desarrollo y crecimiento } \\
\text { utilizando como pretexto el gusano que aparece en las guayabas. }\end{array}$ \\
\hline
\end{tabular}

Fuente: elaboración propia.

Una vez los alumnos respondieron las preguntas en su respectiva bitácora, los 38 registros fueron objeto de análisis, y, finalmente se categorizaron para el análisis de las concepciones (tabla 2).

Tabla 2. Sobre las categorías de análisis halladas y sus definiciones.

\begin{tabular}{|l|l|}
\hline \multicolumn{1}{|c|}{ Categoría } & \multicolumn{1}{|c|}{ Definición y características } \\
\hline Ideas de ciencia vigentes & $\begin{array}{l}\text { Son las ideas encontradas en las expresiones del discurso de los alumnos que coinciden con plan- } \\
\text { teamientos y teorías aceptados por la ciencia actual, y que, sin embargo, requieren clarificación en } \\
\text { la explicación. }\end{array}$ \\
\hline Ideas de ciencia no vigentes & $\begin{array}{l}\text { Son las ideas encontradas en las expresiones del discurso de los alumnos que no coinciden con las } \\
\text { aceptadas por la ciencia actual, corresponden a una ciencia liquidada o caduca, o también, a una } \\
\text { inadecuada interpretación de lo que es considerado ciencia vigente. }\end{array}$ \\
\hline $\begin{array}{l}\text { Ideas de connotación diferentes de las } \\
\text { de desarrollo y de crecimiento biológico }\end{array}$ & $\begin{array}{l}\text { Son las ideas encontradas en las expresiones del discurso de los alumnos que son aceptadas, pero } \\
\text { cuyas explicaciones sobre desarrollo y crecimiento son de connotación diferentes de lo biológico. }\end{array}$ \\
\hline Ideas descontextualizadas & $\begin{array}{l}\text { Son las ideas encontradas en las expresiones del discurso de los alumnos, y en las cuales la secuen- } \\
\text { cia de proposiciones no satisface una coherencia semántica, en razón de su ordenamiento para que } \\
\text { tenga un significado; también, cuando la respuesta no es acorde a la pregunta. Son ideas general- } \\
\text { mente no esperadas. }\end{array}$ \\
\hline
\end{tabular}

Fuente: elaboración propia.

\section{Momento 2. Experiencia con Drosophila melanogaster}

En las 12 sesiones del momento dos, por equipos, los alumnos prepararon medios de cultivo para dar inicio al ciclo de vida de la mosca y así realizar las observaciones sobre su desarrollo y su crecimiento; hicieron registros -dibujos y sus respectivas descripciones - en su guía: ¿Cómo se desarrolla y crece un organismo? experiencia con Drosophila melanogaster, los cuales les sirvieron para discutir con sus compañeros y con el docente, al tiempo que compartieron información relevante por medio de la guía: Me aproximo a eventos de desarrollo y crecimiento con la mosca de la fruta y dos videos sobre su ciclo de vida. Las interacciones discursivas fueron grabadas como audios que luego fueron transcritos; poste- riormente, se seleccionaron fragmentos significativos teniendo en cuenta la notación especializada de Edwards y Potter (1992) y se categorizaron por temas identificados en el discurso -biogénesis, dimorfismo sexual, tipos de reproducción, oviparismo y viviparismo y etapas de desarrollo y de crecimiento-. La interpretación de las concepciones - proposiciones-de los alumnos en los fragmentos significativos se hizo relatando las actividades que promovieron la conversación en su respectiva sesión de clase, pero denotando, principalmente, los significados de las proposiciones de los alumnos, y estos, a su vez, atribuidos de acuerdo con reglas -basadas en los principios de funcionalidad, estructuralidad y referencialidad, además de la coherencia- planteadas por van Dijk $(1985,2007)$ para el análisis semántico del discurso. Finalmente, se establecieron las implicaciones. 


\section{Resultados}

En las tablas 3 y 4 se presenta una muestra representativa de las concepciones iniciales identificadas en el discurso de los alumnos respecto al desarrollo y el crecimiento de un organismo, con su respectivo análisis semántico. Todo ello corresponde al momento 1 de esta investigación.

Tabla 3. Concepciones de los alumnos respecto a la pregunta 1, bajo dos categorías diferentes

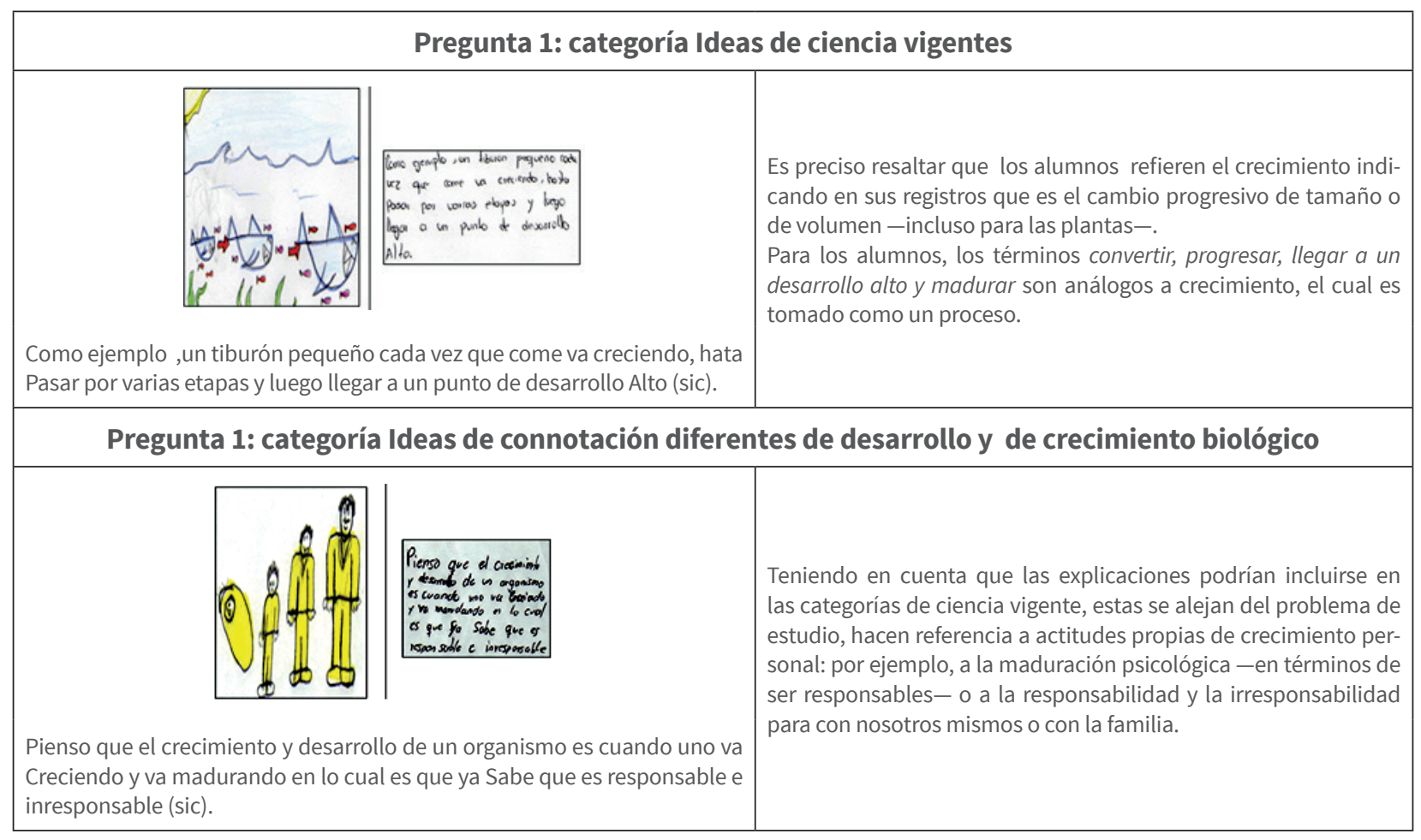

Fuente: elaboración propia.

Tabla 4. Concepciones de los alumnos respecto a las preguntas 2,3 y 4, en sus respectivas categorías.

\begin{tabular}{|c|c|}
\hline \multicolumn{2}{|c|}{ Pregunta 2: categoría Ideas de ciencia no vigentes } \\
\hline 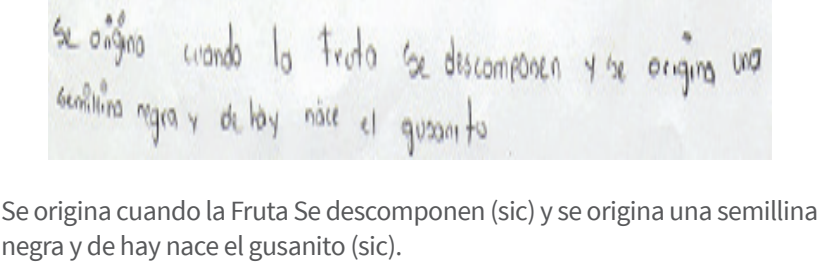 & $\begin{array}{l}\text { Tendencia marcada a desconocer el origen del gusano a partir } \\
\text { de otro preexistente en condiciones naturales, y trayendo en sus } \\
\text { explicaciones ideas de ciencia no vigentes, como la generación } \\
\text { espontánea. }\end{array}$ \\
\hline \multicolumn{2}{|c|}{ Pregunta 3: categorías Ideas de ciencia vigentes } \\
\hline $\begin{array}{l}\text { Es para Depoator sus Huevos para aue tensan } \\
\text { Donde Creser y alimentarce y tener un } \\
\text { ambiente adecuado para su. Desarrollo. }\end{array}$ & $\begin{array}{l}\text { Tendencias: } \\
\text { 1. Alumnos que explican que la mosca llega atraída por los olores } \\
\text { de la guayaba, pero no finalizan la idea. } \\
\text { 2. Alumnos que explican que la mosca llega a comer como razón } \\
\text { obvia. } \\
\text { 3. Tendencia menor: alumnos que visualizan que la fruta en des- } \\
\text { composición es el medio ideal para que la mosca cumpla su ciclo } \\
\text { reproductivo. }\end{array}$ \\
\hline
\end{tabular}




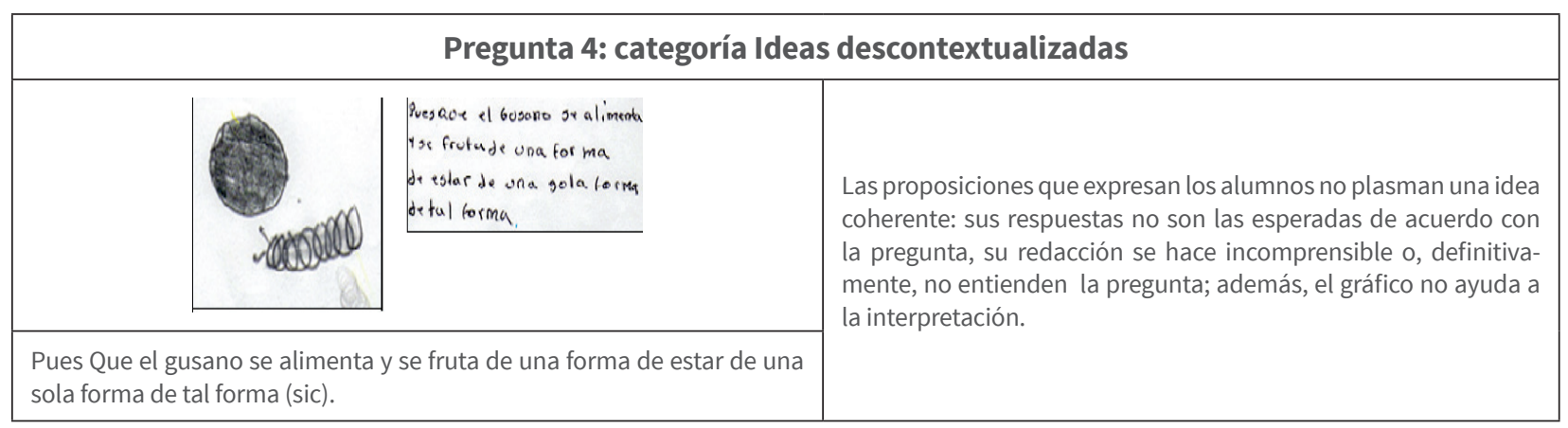

Fuente: elaboración propia.

\section{Síntesis en torno al desarrollo y crecimiento de un organismo}

Los alumnos significan los procesos de crecimiento de un organismo en varios sentidos: el primero y más evidente es el aumento paulatino de tamaño y de volumen de un organismo; el segundo está encaminado a significar el crecimiento como estados de maduración sicológica o como el grado de responsabilidad de una persona ante la sociedad; finalmente, para ellos, el crecimiento puede ser el aumento en el número de bacterias de una colonia.

Aunque hubo dibujos en los que se muestran las etapas de desarrollo de un organismo, como es el caso de la metamorfosis de la mariposa, en general, dentro de su discurso, los alumnos no significaron una explicación coherente respecto a los procesos ocultos que suceden en la transformación de un organismo desde el huevo fecundado hasta el estado adulto.

Respecto a la presencia de un gusano dentro de la guayaba, muy pocos de los alumnos afirmaron que provenía de un huevo, como consecuencia de una fecundación, sin ir más allá, para explicar el ciclo reproductivo completo. Las explicaciones simples dan cuenta de que el gusano está presente en el fruto, simplemente, porque llegó allí o hace parte de esta, haciendo alusión inconsciente a la generación espontánea.

En términos generales, las concepciones que tienen los alumnos acerca del crecimiento y el desarrollo de un organismo se vislumbran a la luz de su propia experiencia; la intención de sus explicaciones es a partir de la vivencia con su propio entorno y manifestándolo en su lenguaje común. Desconocen los procesos de desarrollo en un organismo, porque, para ellos no son visibles ni manipulables.

\section{Síntesis en torno a las causas que pudieron originar estas concepciones}

La primera causa corresponde al sentido común, a las ideas de los alumnos que derivaron de la observación primaria, la cual es tangible a través de los sentidos desde el entorno. Este tipo de concepciones inadecuadas tienen un alto grado de veracidad, son muy arraigadas, $y$, por tanto, difíciles de tratar (Carrascosa, 2005), al hacer parte de su propia representación del mundo físico del que elaboran hipótesis y teorías (Candela, 1999), o bien, porque provienen de las experiencias, los lenguajes y los conocimientos nacidos de algo que constituye la relación global entre el humano y el mundo que lo rodea desde temprana edad (Arcá et al., 1990).

La segunda causa estuvo relacionada con la confusión de conceptos o una interpretación diferente de la de los planteamientos y las teorías aceptados por la ciencia. Estas concepciones se relacionan, principalmente, con el modo de pensar espontáneo del alumno; sin embargo, también pueden deberse a dificultades en la metodología utilizada en el proceso de enseñanza y aprendizaje de las ciencias naturales, originadas, entonces, en el contexto escolar (Carrascosa, 2005). Al respecto, se dan cuatro casos: a) cuando el docente que enseña tiene el mismo nivel de concepciones de sus alumnos, por lo que no puede ayudar a sus alumnos a adecuarlas; b) cuando en la didáctica utilizada se ignoran las concepciones inadecuadas de los alumnos, las cuales son obstáculos u oportunidades para el aprendizaje; c) cuando la estrategia metodológica de años escolares anteriores no fue apropiada y dejó vacíos conceptuales, que ahora originan la confusión en el alumno, y d) cuando el docente desconoce la historia - progresos, cambios, controversias- de cierto conocimiento científico, cuestión que, en caso de tenerse en cuenta, puede ayudar a comprender las concepciones inadecuadas de los alumnos, dada su similitud con la 
ciencia que estuvo vigente a lo largo de algún periodo de la historia de la ciencia (Carrascosa, 2005), y así diseñar estrategias didácticas para su adecuación.

Una tercera fuente de las concepciones inadecuadas identificadas en los alumnos es atribuida a la información extraescolar proveniente de: a) el lenguaje, oral o escrito, de otras personas con concepciones similares, y b) los medios de comunicación, y que puede ser malinterpretada por el alumno, o la pueden difundir, a veces, con significados diferentes de los de la ciencia aceptada. La cuarta y última causa es la incomprensión lectora o escritural de los alumnos, manifestada en las respuestas que no tuvieron relación con el contexto dado; ello deriva en plasmar ideas incoherentes - llamadas descontextualizadas en esta investigación- o que fueron incomprensibles en su significado porque no hubo una secuencia ordenada entre las proposiciones o las palabras que lo constituyen (van Dijk, 1985, 2007). También es posible que hayan sido dadas de manera espontánea, en su afán por responder, o que no existan concepciones en torno al problema de estudio porque no ha habido experiencias escolares o extraescolares significativas.

Respecto al momento 2 , se presenta enseguida la atribución de significados a las proposiciones - concepcionesde los alumnos teniendo en cuenta de manera integrada las reglas para el análisis semántico del discurso planteadas por van Dijk $(1985,2007)$ en los fragmentos de conversaciones seleccionadas del discurso oral que resultó de 12 sesiones de clase, donde los alumnos, junto con el docente, interactuaron sobre el desarrollo y el crecimiento de los organismos a partir de la experiencia con el ciclo de vida de Drosophila melanogaster. Los fragmentos significativos fueron ajustados en su transcripción utilizando el sistema de notación especializada propuesto por Edwards y Potter ${ }^{5}$ (citados en Candela, 2006); además, están orga-

5 Notación especializada: Mo: maestro; Ma: Maestra; Aa 2: Alumna (en este caso la número dos); Ao: Alumno; As: Varios alumnos simultáneamente; $\wedge$ : Indica elevación de la entonación; /: Indica caída de la entonación; =>: Indica frase significativa para el análisis; ${ }^{\circ}$ : Indica un pasaje de habla más bajo en intensidad que el habla adyacente; MAYUS: Indica un pasaje que habla con mayor intensidad que el habla adyacente; *: Indica ruido de fondo no distinguible de las alumnas(os) hablando entre ellas; **: Indica ruido de fondo de mayor intensidad; $><$ : Indica un pasaje que habla más rápido que el circundante; <>: Indica un pasaje que habla más lento que el circundante; [:Indica habla sobrepuesta; :::: : Indica elongación del énfasis en una letra; Subr: Indica énfasis especial dentro de la frase; ((it)): Comentarios del transcriptor, generalmente observaciones sobre el contexto de habla; (2): Pausa medida en segundos, dos en este caso; (.):Pausa perceptible, pero muy corta para medirse en décimas de segundos; =: Habla ligada a la anterior sin el lapso habitual en las conversaciones. nizados según contenidos temáticos identificados que corresponden a la categorización hallada: biogénesis, dimorfismo sexual, tipo de reproducción sexual y asexual -cópula y fecundación-, oviparismo y viviparismo, etapas de desarrollo y crecimiento. Al final, se presentan las implicaciones sobre la enseñanza de este contenido, las cuales fueron derivadas de los significados atribuidos.

\section{Biogénesis}

1. Mo: ¿pero y esos gusanitos de dónde habrán salido? ((los alumnos estaban observando el cultivo base con la mosca de la fruta y discutiendo entre ellos))

2. => Aa1: esos gusanitos van a salir de (1) de::: los olo::res de las bacterias, de lo que se está descomponiendo

3. Mo: entonces (.) ¿Los gusanitos aparecen de la descomposición de la guayaba?

4. Aa1: Sí:

En la línea 2 del fragmento, la palabra salir, en su proposición, significa, para el alumno, en el contexto de la conversación, un sinónimo de nacer; es decir, como si los gusanos emergieran espontáneamente de los olores que provocan las bacterias al descomponer la fruta del cultivo, lo que reafirma la teoría no vigente de la generación espontánea. No obstante, en una anterior discusión sobre la reproducción sexual en la misma sesión, los alumnos habían logrado establecer que la presencia de huevos en el medio de cultivo implicaba los procesos consecutivos de cópula y fecundación bajo la presencia de moscas macho y hembra.

\section{Dimorfismo sexual}

10. Mo: ^¿Listos? (2) ¿alguien ya vio algo? ((Los alumnos en el estereoscopio tenían dos ejemplares, macho y hembra de la mosca, previamente seleccionados por el docente y alumnos; además, tenían dibujos desde la guía 3 de su bitácora y una imagen real ampliada en el televisor desde un microscopio para hallar la diferencia)).

11. =>Ao6: La hembra tie:::ne ((el alumno no continúa su respuesta))

12. => Ao1: La mosca de los órganos del abdomen que es más grande que el otro.

13. Mo: [¿Tú viste la diferencia en el abdomen de las moscas?

14. As: =^Sí

15. Mo: ¿CUÁL VISTE MÁS GRANDE?

16. Ao6: Hay un abdomen >más grande que el otro<.

17. Mo: ^Perfecto 
18. $=>$ Ao1: $>$ la mujer $<$

19. Mo: ¿La mujer?

20. Ao1: La muje::::r tiene el rabo más grande y::: (.) el mosco el rabo más chiquitico y espichadito.

21. Mo: Escuchen lo que está diciendo Bryan (1) Bryan repita para todos.

22. ${ }^{\star \star}=>$ Ao1: que la mujer tiene rabo más grande (.) tiene el abdomen más grande y largo y::: el mosco tiene el rabo como espichadito y pequeño. ((risas))

23. Mo: > ¿SABEN POR QUÉ ESO ES ASí?<

24. => Ao2: porque la hembra necesita el abdomen más gra:::nde para los huevos

25. Mo: <Bie:::n>

Un alumno propuso, desde su pensamiento antropocentrista, la relación entre las caderas de una mujer con el abdomen de la mosca, para lo cual utiliza el término "rabo" - líneas 18 y 20-; tanto así, que se refiere a la mosca como una mujer, y no como una hembra: este último es el término correcto para referirse a los animales, tal como lo hizo el alumno de la línea 11. En efecto, en ambos casos, los alumnos referenciaron el dimorfismo sexual de la mosca "la mujer tiene el rabo más grande y el mosco el rabo más chiquitico y espichadito" - línea 20-. A pesar de que en este fragmento se evidencia cómo los alumnos cumplieron con su objetivo de diferenciar macho de hembra expresándolo con su lenguaje cotidiano; es importante resaltar la analogía que se hizo al referirse a la amplitud del abdomen de la mosca hembra comparándolo con el de una mujer, y al deducirse así que es utilizado para la misma función en la reproducción -línea 24-; es decir, alojar los huevos o al futuro bebé, respectivamente.

\section{Tipos de reproducción asexual y sexual -cópula y fecundación-}

115. Mo: ¿y cuál es la reproducción a:::sexual?

116. As:^cuando proviene solamente de un individuo

117. Mo: si una mosca pone un huevito y esa mosca jamás tuvo un macho ${ }^{\wedge}$ ¿Es posible que salga otra mosca?

118. => Ao1: No:: porque se necesita de los dos (2) macho y hembra (.) y en la reproducción asexual se necesita de uno (.) como en las plantas (4) que se reproducen por sí solas

119. Mo: ${ }^{\wedge}>$ ¿Cómo en las plantas?<

120. => Ao1: Si::::: que se reproducen por la::: fotosíntesis

Ante la respuesta correcta del grupo de alumnos - línea 116-, el docente continúa indagando al respecto con una situación problema -línea 117 - y la respuesta siguiente muestra coherencia y es convincente en su primera parte, ya que subyace la diferencia entre los dos tipos de reproducción -línea 118-, pero en la segunda parte crea en el docente una reacción que lo obliga a preguntar con la misma respuesta del alumno, y la réplica es aún más interesante, pues da a entender que la reproducción asexual es como sucede en las plantas a través de la fotosíntesis -línea 120-.

\section{Oviparismo y viviparismo}

1. Aa5: profe una pregunta (.) ¿A la mosca se le rompe la fuente como a una mujer? ((risas de los alumnos))

2. Mo:<que:: si a una:: mosca se le ro:::mpe la:: fuente igual que a una mujer>

3. Mo: =esa pregunta yo sé que tú::: (.) tú la sabes ((el docente señala a un alumno))

4. Ao6: si:: puede ser que la sepamos (.) pe:::ro::: (.) $>$ claro que no $<$ (.) > no rompe fuente $<$

5. *Mo: no rompe fuente! ¿Por qué? ((risas de los alumnos)) (3)

6. ${ }^{\star \star}=>$ Ao6: PORQUE SON HUEVOS ¿NO? ((Se escucha entre los alumnos los términos ovíparos y vivíparos))

7. ${ }^{\star \star}=>$ Ao6: =e:::s una::: incubación (1) son huevos (3)

En las líneas 4, 6 y 7 del fragmento, el alumno seleccionado por el docente da cuenta de que la mosca "no rompe fuente" al poner huevos que son luego incubados, de modo que supone implícitamente un nacimiento ovíparo que no requiere parto; es decir, un nacimiento desde el huevo puesto por la mosca -evidencia de una relación causa- consecuencia entre los hechos-. Para el caso de la mosca, incubar no es un hecho posible. Aunque el alumno generaliza el término incubar, debido a sus experiencias de sentido común, este no representa obstáculo para lo que se pretende en la conversación...

\section{Etapas de desarrollo y crecimiento}

9. Mo: ¿Para qué eran esos medios de cultivo?

10. Ao1:pue::s (.) para que las moscas se alimentaran

11. Mo: ¿Para que las moscas se alimentaran? (1) ¿Solamente para que las moscas se alimentaran?

12. Ao1: Para que ovoposicionaran

13. Mo: ¿Y qué es ovoposicionar?

14. Ao1:/¿Cómo pariar?

15. Mo: ^¿Qué es pariar?

16. Aa2: >Cuando la hembra coloca el huevo sobre la guayaba<

17. Mo: Entonces el medio de cultivo sirve para ovoposicionar ¿y para qué más?

18. ${ }^{\circ} \mathrm{Aa3}$ : para alimentarse 
19. Mo: ¿Para alimentarse ^quién?

20. Aa3: la mosca

21. Mo: ¿La mosca? (.) ¿Seguro?

22. => Ao4: [Y la larva para alimentarse en sus tres estados larvarios

23. Mo: ¿De dónde salen esos tres estados larvarios?

24. => A04: las larvas salen de los huevos (.) y:: ellas se alimentan de la guayaba (.) >y va a crecer< (.) Después obtiene todos los nutrientes y se convierte en pupa y:: (.) de ahí sale el adulto

Analizando globalmente el fragmento, es interesante identificar la coherencia semántica de la conversación, donde los alumnos participantes hacen referencia al medio de cultivo como ese sustrato - la guayaba madura- del cual la mosca, en su estado adulto, no solo se alimenta -líneas 10 y 18 -sino que, además, lo utiliza para ovoposicionar -líneas 12 y 16 -, y luego, para que las larvas nacidas de esos huevos puedan alimentarse y aumentar de tamaño atravesando sus tres estados larvarios; es decir, para que crezcan, tal como lo detallaron en la experiencia. El alumno de las líneas 22 y 24 logra establecer una proposición posible dentro del marco de hechos aceptados por la ciencia para dar cuenta de las transformaciones - secuencia de etapas-que suceden en el ciclo de vida de la Drosophila melanogaster, y así logra comunicar el orden: huevo, larva, pupa y mosca adulta; además, relaciona la guayaba en el cultivo como el alimento que le permite a la larva desarrollarse y crecer, y mejor aún, cuando enseguida menciona en su proposición "después obtiene todos los nutrientes, y se convierte en pupa", significando, probablemente, para el alumno, que la larva es muy activa y debe alimentarse ávidamente para almacenar los nutrientes necesarios que le permitirán su subsistencia cuando se transforme -sinónimo de "se convierte" en la frase del alumno-al estado de desarrollo siguiente. En la proposición de la línea 24, el alumno utiliza el término "salen" para expresar que las larvas nacen cuando el huevo eclosiona -significado que se ha atribuido a este término en varias oportunidades-.

El alumno 1 de la conversación, en la línea 14, confunde ovoposicionar con el acto de parir - "pariar" en la conversación-, y así establece una comparación entre el acto de parir de un vivíparo con la ovoposición de la mosca; sin embargo, sabemos que en los organismos con nacimiento ovíparo parir no es un hecho posible, por lo cual él mismo duda de su proposición. No obstante, en la línea 16 una alumna da un significado adecuado al morfema representado en la expresión "la hembra coloca el huevo sobre la guayaba", queriendo decir que hay una postura de un huevo.

\section{Implicaciones sobre la enseñanza de la situación de estudio}

Sobre los gusanos en la guayaba -larvas observadas-, los alumnos establecieron que su presencia estuvo relacionada con la postura de huevos fecundados -ovoposición-realizada por una mosca, y que estos, con el tiempo, se transformaban en gusanos; de este modo, aseguraron que previamente debió de suceder en el cultivo la cópula, y reconocieron así la reproducción sexual de estos insectos. Sumado a ello, en sus proposiciones los alumnos dieron cuenta de que los gusanos observados correspondían a la fase larvaria del ciclo de vida de la mosca de la fruta, por lo cual infirieron que las nuevas moscas adultas dentro del frasco, que se generaban a partir de estos, eran de la misma especie de sus progenitores. Finalmente, los alumnos rebatieron la generación espontánea reconociendo que la función de la guayaba madura fue proporcionar nutrientes para promover el ciclo de vida objeto de estudio, razón por la cual las moscas hembras son atraídas por sus olores, y sobre dicha fruta, ovoposicionar.

Sobre el dimorfismo sexual de la mosca Drosophila melanogaster, implícitamente lo mencionaron, al proponer diferencias físicas entre ambos: una de ellas fue observar el amplio tamaño del abdomen de la mosca hembra en relación con el del macho. Otra diferencia identificada fue la forma del abdomen, cuya terminación en el caso de la mosca hembra es "en punta"; sin embargo, no relacionaron esta terminación con la presencia del oviscapto para efectos de la ovoposición.

Para que los alumnos referenciaran situaciones relacionadas con los tipos de reproducción sexual y asexual, así como con la fecundación, inevitablemente, debieron acudir a una cópula previa, que, para el caso de la sexual, involucró dos progenitores - mosca macho y mosca hembra-. Los significados dados a las concepciones de los alumnos sobre reproducción sexual evidenciaron inicialmente la presencia de estructuras reproductivas en ambos progenitores, las que al complementarse originan un nuevo organismo; luego, dichas estructuras tuvieron relación con los gametos sexuales: espermatozoide y óvulo. Además, reconocieron que los espermatozoides son aportados por el macho, los cuales son introducidos en la hembra durante la cópula, para una posterior fecundación interna; sin embargo, piensan que el espermatozoide se introduce completo en el óvulo. Los huevos ovopositados en el cultivo, y que luego se transformaron en larvas, estuvieron relacionados con la idea de la fecundación de tipo interno, mencionada por los alumnos, quienes, además, explicaron dicho momento como el restablecimiento de la carga cromosómica en 
organismos diploides - por ejemplo, la mosca objeto de estudio- a partir de la unión de gametos con la mitad del número de cromosomas - condición haploide-.

Sobre las formas de nacimiento, los alumnos, al establecer una analogía entre la Drosophila melanogaster del cultivo con la mujer - humanos-, significaron la viviparidad como un desarrollo del individuo dentro del vientre y luego el nacimiento del bebé, y la oviparidad, como un desarrollo dentro de un huevo ovopositado previamente -desarrollo externo-, para que, luego de un tiempo, ocurra el nacimiento de la larva. Teniendo en cuenta las condiciones de viviparidad y oviparidad, los alumnos propusieron que para la mosca - ovípara-contrariamente a la mujer - vivípara-, no existe la posibilidad de un embarazo, ni de, por consiguiente, presentar parto; también, que la mosca no incuba sus huevos, como lo han observado en algunas aves - principalmente, domésticas-, ni, menos aún, cría a sus hijos.

La observación detallada y sistemática del ciclo de vida de Drosophila melanogaster en los cultivos les permitió a los alumnos dar cuenta del ordenamiento de los estadios y las transformaciones sobre el desarrollo y el crecimiento que ocurren: cópula, fecundación interna, huevos, ovoposición, larva, pupa y mosca adulta. Respecto a los procesos de desarrollo que sucedían a escala interna, hubo confusión a la hora de explicarlos, por lo cual los referenciaron de manera sencilla como el desarrollo en el interior de la pupa que permite la formación de sus órganos - alas, ojos, etc- para transformarse hasta el estado de mosca adulta.

El término salir fue empleado en las concepciones de los alumnos para referirse a varias situaciones según el contexto del habla; en tal sentido, fueron varios los significados atribuidos, por ejemplo, cuando decían "los gusanos salen de la descomposición" o "que de dos individuos salga uno", se referían a nacer -contexto: origen de un organismo-; cuando proponían la idea "las dos se forman adentro, solo que una sale como en huevo", claramente, la que sale como en huevo es la forma ovípara, y, por tanto, el término fue sinónimo de ovoposicionar -contexto: formas de nacimiento-; y cuando lo conceptualizaron en la idea "las larvas salen de los huevos" - contexto: estadios de desarrollo y crecimiento-, ello se refirió a las transformaciones de un huevo hasta larva; además, puede ser sinónimo de eclosionar. El docente también promovió la utilización del término.

Las concepciones en el discurso de los alumnos se caracterizaron por ser legitimadas directa e inmediatamente por lo que observaron en el cultivo sobre el ciclo de vida de la mosca Drosophila melanogaster; por ello, con veraci- dad y sin repetir lo que la ciencia reducía en la información suministrada durante la experiencia o la encontrada en un libro de texto, los alumnos pudieron pensar y comunicar. Si bien los conceptos que lograron significar tuvieron una conexión directa con la experiencia vivida, sus definiciones en el discurso no fueron exactas, pero sí próximas a lo que transcurría en el fenómeno. Caso contrario cuando los alumnos proponían ideas sobre los procesos de desarrollo que se dan a escala celular o interna -organogénesis, mitosis y meiosis, entre otros-, pues al no haber una experiencia tangible o sensorial que nutriera el entendimiento de dichos objetos de estudio, hubo confusión en sus explicaciones, o, simplemente, repetición de la información tal cual, sin apropiarla.

Los alumnos propiciaron contextos discursivos desde sus experiencias de sentido común; en su mayoría, valiéndose de explicaciones construidas desde el antropocentrismo. Es así como en una de las conversaciones aparecen las siguientes concepciones: "que la mujer tiene rabo - cadera- más grande, tiene el abdomen más grande y largo", y más adelante, "porque la hembra - mosca- necesita el abdomen más grande para los huevos", lo cual evidencia la relación entre las caderas amplias de las mujeres con el abdomen de mayor tamaño en la mosca hembra, y los lleva a inferir que son de ese modo, que, funcionalmente, sirven para lo mismo: alojar el bebé para el caso de la mujer, y mantener temporalmente los huevos en el caso de la mosca hembra. Otro ejemplo respecto al uso de este tipo de analogías fue el caso de la pregunta: “¿A la mosca se le rompe la fuente, como a una mujer?", y a partir de esta, inferir que en los organismos ovíparos, como la mosca, no existe la posibilidad de un embarazo que conllevaría el rompimiento de fuente, como sucede en los vivíparos.

En las discusiones entre alumnos surgieron explicaciones diferentes, lo que indicó pensamientos y concepciones distintas; sin embargo, a través de la confrontación con el docente y los demás compañeros, y mediante las consultas realizadas en torno a la experiencia de aula, pudieron adquirir coherencia lógica y resignificar favorablemente sus ideas. Sobre este punto, las preguntas del docente para propiciar la confrontación no deben generar confusión ni inducir a la respuesta, como se evidenció en algunas conversaciones, sino, por el contrario, hacer que sus alumnos piensen y expresen sus ideas, que duden, que hagan comentarios, que reflexionen y logren resolver llegando a conclusiones.

\section{Conclusiones}

Las respuestas rápidas mediadas por el sentido común y la confusión de conceptos y de planteamientos - por 
dificultades con la didáctica utilizada en años anteriores, por información proveniente de los medios de comunicación y de personas de su entorno, por las ideas descontextualizadas y por la falta de experiencias significativasfueron las posibles causas de las concepciones iniciales identificadas en el discurso de los alumnos.

Las concepciones sobre el origen del gusano en la guayaba, en la mayoría de los alumnos, tuvieron similitud con la teoría de la generación espontánea; en ese sentido, desconocieron o confundieron procesos de desarrollo y de crecimiento que suceden durante el ciclo de vida de Drosophila melanogaster referenciando que el gusano utiliza la fruta para vivir o alimentarse de ella, y así "desarrollarse". También, para entonces, los alumnos explicaron sin dificultad en su discurso el crecimiento de un organismo - por ser un proceso tangible y verificable por los sentidos-, como un cambio en el volumen y el tamaño; mientras, hubo confusión para dar cuenta de los fenómenos no observables a simple vista o que suceden en el ámbito interno, como el desarrollo en sí del organismo.

Aplicados al discurso bilógico, los planteamientos y las reglas propuestas por van Dijk para el análisis semántico permitieron: a) identificar proposiciones -concepciones-, de los alumnos, generadas a partir de la interacción discursiva alumno-alumno o alumno-docente, las cuales tenían intención de dar respuesta a los procesos que sucedían paulatinamente durante la observación del ciclo de vida de la mosca de la fruta; b) analizar aspectos sobre la coherencia semántica del discurso reconociéndolo no solo como un conjunto de oraciones, sino como una secuencia ordenada de proposiciones subyacentes para que fuera aceptable, $y$, a partir de esto, seleccionar fragmentos de audio transcritos, para categorizarlos según contenidos temáticos identificados sobre el contenido de interés, y c) atribuir significados intencionados por parte del docente investigador a una secuencia de proposiciones -fragmento-, cumpliendo tres requisitos concurrentes: que las palabras que componían una expresión, según el contexto, tuvieran significado en la base de sus proposiciones; que las secuencias de proposiciones mantuvieran relación con proposiciones subyacentes acordes al contexto, $y$, finalmente, asignar valores de verdad a las proposiciones individuales dentro del mismo contexto teniendo en cuenta el discurso como un todo.

Convertir una situación cotidiana, como la presencia de moscas alrededor de una guayaba o de gusanos en su interior, es un espacio problemático, que motivó a los alumnos a emprender el camino hacia la comprensión del ciclo de vida de Drosophila melanogaster; para eso, la observación sistemática y detallada que hicieron de los eventos que sucedían en el cultivo de estudio permitió discutirlos con los demás sujetos y corroborarlos con los planteamientos y las teorías sancionadas. De este modo, lograron resignificar y complejizar sus ideas. Por eso, más allá de las explicaciones simplistas, reduccionistas - y hasta equivocadas-, que se le dan cotidianamente al gusano que en ocasiones aparece al abrir una guayaba -incluso considerarlo un hecho aislado del ciclo de vida de la mosca de estudio-, en la clase pasó a ser un problema para el conocimiento y la comprensión de los procesos relacionados con el desarrollo y el crecimiento de los organismos, $\mathrm{y}$, particularmente, de este díptero, a través de distintas rutas explicativas propuestas por los alumnos, para así darle un sentido renovado a la situación cotidiana.

A partir de la atribución de significados hecha, se reveló la resignificación de concepciones respecto a las del primer momento, por cuanto se hizo evidente que los alumnos en su discurso enunciaron proposiciones sobre desarrollo y crecimiento más operativas y más próximas a lo que sucede durante el ciclo de vida de la mosca de la fruta empleada. Ejemplo de ello es que la generación espontánea fue resignificada por la teoría de la biogénesis, lo cual implica que sus explicaciones en las discusiones referenciaron conceptos como dimorfismo sexual, reproducción sexual y asexual, cópula, fecundación, viviparismo y oviparismo, ovoposición y sobre los estadios de desarrollo y crecimiento de un organismo.

La intervención del docente en las discusiones de clase condujo a los alumnos a buscar la reflexión con sus constantes preguntas, a propiciar comentarios, a fijar tiempos, a introducir dudas, a confrontar explicaciones, a crear conflictos, a brindar la información pertinente cuando ameritaba hacerlo, o ayudar a que la buscaran, y hacer que llegaran a sus propias conclusiones. De manera indirecta, el docente apoyó que los alumnos construyeran y reconstruyeran conocimiento a partir de sus concepciones iniciales confrontadas con una experiencia palpable.

El antropocentrismo fue la base para la construcción de proposiciones coherentes por parte de los alumnos en los dos momentos de esta investigación; fue así como los condujo a establecer semejanzas y diferencias entre los procesos de desarrollo y crecimiento de especies comparando situaciones a partir de su vivencia, para asignarlos a otros organismos. Tal es el caso de llegar a pensar en el embarazo y el parto de la mosca de la fruta, tal como ocurre en las mujeres; incluso, que este insecto cría a su progenie de manera similar a la de los humanos, así como dar cuenta del crecimiento de organismos con las etapas de desarrollo de los humanos, o comparar las caderas de la mujer con el abdomen de las moscas. Sin embargo, a partir de dichas comparaciones, "también analogías", el alumno pudo complejizar sus concepciones durante 
las discusiones, al contrastarlas con el discurso biológico legitimado.

Las analogías empleadas por los alumnos en ambos momentos fueron una habilidad de pensamiento importante en la resignificación de sus concepciones. En este sentido, por medio de la habilidad para comparar sus representaciones mentales previas con una situación problema - para este caso, el desarrollo y el crecimiento de un organismo-, en su imaginario se formaron, desde las actividades culturales, nuevas concepciones que permitieron explicaciones más próximas al discurso biológico existente; de esta forma, por ejemplo, dieron cuenta de la condición de reproducción de la mosca de la fruta diferenciándola de la viviparidad de una mujer y asemejándola con la oviparidad de una mariposa.

La experiencia con Drosophila melanogaster fue el evento que nutrió la imaginación, la memoria y la sensibilidad de los alumnos; esta fue no solo un simple acercamiento a la realidad, sino un acontecimiento que impulsó al alumno a escribir y a confrontar su experiencia de crecimiento y desarrollo, mediante un lenguaje evidente en un discurso autónomo, en el cual manifestó los avances de su propio proceso cognitivo resignificando paulatinamente sus concepciones iniciales.

El método de enseñanza activo empleado en este trabajo de investigación dio la oportunidad para que el alumno, en torno a las experiencias de aula vividas con Drosophila melanogaster, fuera el artífice de su propio conocimiento, mediante la actividad grupal, donde cada alumno expuso sus concepciones, disertó con sus pares y resignificó sus saberes; así mismo, el docente enriqueció su práctica pedagógica al mediar, indagar, guiar y aceptar un espacio para notar sus errores, enmendarlos, y, de esa forma, reivindicar su quehacer educativo. Así, se vislumbró un discurso pedagógico, mediante un proceso dialógico intersubjetivo, expresado en el intercambio de concepciones, basado, a su vez, en un modelo didáctico que en últimas buscó un aprendizaje tanto para los alumnos como para los docentes.

El proceso investigativo facilitó que los alumnos resignificaran sus saberes mediante la experiencia de aula; es decir, sus concepciones iniciales fueron transformándose en la medida en que confrontaban el ciclo de vida de Drosophila melanogaster con la información adquirida a través de la interacción discursiva. Para los docentes investigadores, se constituyó en una experiencia en la cual se evidencia que es necesario disponer de las concepciones previas que poseen los alumnos, así como su edad y sus ritmos de aprendizaje; todo ello, para abordar cualquier tema de ciencias en general.
Las concepciones identificadas en el discurso de los alumnos y los significados atribuidos a estas en el presente documento son un poderoso recurso que debe tomarse en consideración cuando se pretende enseñar contenidos sobre el desarrollo y el crecimiento de los organismos en el nivel de octavo grado, pues son una explicación aproximada a la realidad del fenómeno, $y$, en ese sentido, son susceptibles de ser resignificadas, modificadas o tratadas con nuevas experiencias.

\section{Referencias}

Arcá, M., Guidoni, P. y Mazzoli, P. (1990). Enseñar ciencia. Cómo empezar: reflexiones para una educación científica de base. España: Paidós.

Audesirk, G., Audesirk, T. y Byers, B. (2008). Biología. La vida en la tierra. México Pearson.

Campbell, N., Mitchell. L. y Reece, J. (2001). Biología, conceptos y relaciones. México: Pearson.

Candela, A. (1991). Argumentación y conocimiento científico escolar. Infancia y Aprendizaje, 55, 13-28.

Candela, A. (1999). Prácticas discursivas en el aula y calidad educativa. Revista Mexicana de Investigación Educativa, 4, 273- 298.

Candela, A. (2006). Del conocimiento extraescolar al conocimiento científico escolar: Un estudio etnográfico en aulas de la escuela primaria. Revista Mexicana de Investigación Educativa, 11, 797-820.

Carrascosa, J. (2005). El problema de las concepciones alternativas en la actualidad, parte I, análisis de las causas que las originan y/o mantienen. Revista Eureka, 2, 183-208.

Curtis, H., Barnes, N., Schnek. A. y Massarini. A. (2011). Biología. Madrid: Editorial Médica. Panamericana.

van Dijk, T. (1985). Análisis del discurso semántico. Manual de Análisis del Discurso, 2 (pp. 103-136). London: Academic Press.

Van Dijk, T. (2007). Estructuras y Funciones del Discurso. Edición actualizada. Traducción de: Myra Gann, Martí Mur y Josefina Anaya. México: Siglo XXI.

Enciclopedia del estudiante. (2006). Tomo 1: Ciencias de la vida. Buenos Aires: Santillana.

Giordan, A. y De Vecchi, G. (1995). Los orígenes del saber: de las concepciones personales a los conceptos científicos. España: Díada editora.

Kimball, J. (2001). Biología. Addison-Wesley. Bogotá: Iberoamericana.

Starr, C., y Taggart, R. (2004). Biología. La unidad y diversidad de la vida. México, D.F.: Thompson. 